\title{
A transport equation for reaction rate in turbulent flows
}

\author{
V. A. Sabelnikov, ${ }^{1}$ A. N. Lipatnikov, ${ }^{2, a)}$ N. Chakraborty, ${ }^{3}$ S. Nishiki, ${ }^{4}$ \\ and T. Hasegawa ${ }^{5}$ \\ ${ }^{1}$ ONERA-The French Aerospace Laboratory, F-91761 Palaiseau, France \\ ${ }^{2}$ Department of Applied Mechanics, Chalmers University of Technology, \\ Gothenburg 412 96, Sweden \\ ${ }^{3}$ School of Mechanical and Systems Engineering, Newcastle University, \\ Newcastle Upon Tyne NE1 7RU, United Kingdom \\ ${ }^{4}$ Department of Mechanical Engineering, Kagoshima University, \\ Kagoshima 890-0065, Japan \\ ${ }^{5}$ Institute of Materials and Systems for Sustainability, Nagoya University, \\ Nagoya 464-8603, Japan
}

(Received 10 June 2016; accepted 22 July 2016; published online 3 August 2016)

\begin{abstract}
New transport equations for chemical reaction rate and its mean value in turbulent flows have been derived and analyzed. Local perturbations of the reaction zone by turbulent eddies are shown to play a pivotal role even for weakly turbulent flows. The mean-reaction-rate transport equation is shown to involve two unclosed dominant terms and a joint closure relation for the sum of these two terms is developed. Obtained analytical results and, in particular, the closure relation are supported by processing two widely recognized sets of data obtained from earlier direct numerical simulations of statistically planar 1D premixed flames associated with both weak large-scale and intense small-scale turbulence. Published by AIP Publishing. [http://dx.doi.org/10.1063/1.4960390]
\end{abstract}

The critical point of turbulent reacting flow theory stems from averaging reaction rates subject to fluctuations in the local temperature, $T$, and concentrations. The problem is particularly severe in the case of premixed burning, because (i) the rates of reactions that control heat release depend non-linearly on $T$, (ii) the magnitudes of the temperature fluctuations are typically large, and (iii) these fluctuations exhibit a wide range of length and time scales. As reviewed elsewhere, ${ }^{1-6}$ models developed to solve this highly non-linear and multiscale problem can be divided into two major groups: (i) models that yield a closed expression for the mean reaction rate, and (ii) models that deal with a transport equation for a quantity, e.g., mean scalar dissipation rate, ${ }^{6,7}$ or mean flame surface density, ${ }^{3,4,8-10}$ that is assumed to be linearly related with the mean reaction rate. However, the present authors are not aware of a transport equation derived directly for a mean reaction rate in a turbulent flow. The goal of the present communication is to fill this gap by introducing such a transport equation and exploring it in the case of premixed mode of burning.

To derive a transport equation for reaction rate, let us assume that the state of a mixture in a premixed flame is characterized by a single combustion progress variable $c$, which varies from zero in unburned gas to unity in combustion products. Such a simplification is typical for a majority of models that address adiabatic burning at low Mach and unity Lewis numbers. ${ }^{4}$ The transport equation of $c$ takes the following form:

$$
\rho \frac{\partial c}{\partial t}+\rho \mathbf{u} \cdot \nabla c=\nabla \cdot(\rho D \nabla c)+\rho W
$$

where $t$ is time, $\mathbf{u}$ is the flow velocity vector, $\rho=\rho(c), D=D(c)$, and $W=W(c)$ are the density, molecular diffusivity, and reaction rate, respectively. The four terms in Eq. (1) are associated with non-stationarity, convection, molecular diffusion, and reaction, respectively.

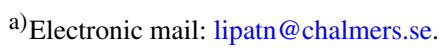


Then, using the continuity equation

$$
\frac{\partial \rho}{\partial t}+\nabla \cdot(\rho \mathbf{u})=0
$$

we have

$$
\begin{gathered}
\frac{\partial}{\partial t}(\rho W)+\nabla \cdot(\rho \mathbf{u} W)=\rho \frac{\partial W}{\partial t}+\rho \mathbf{u} \cdot \nabla W=\frac{d W}{d c}\left[\rho \frac{\partial c}{\partial t}+\rho \mathbf{u} \cdot \nabla c\right] \\
=\frac{d W}{d c} \nabla \cdot(\rho D \nabla c)+\rho W \frac{d W}{d c}=\nabla \cdot\left(\rho D \frac{d W}{d c} \nabla c\right)-\rho D \nabla c \cdot \nabla\left(\frac{d W}{d c}\right)+\rho W \frac{d W}{d c} .
\end{gathered}
$$

Finally, we arrive at

$$
\frac{\partial}{\partial t}(\rho W)+\nabla \cdot(\rho \mathbf{u} W)=\nabla \cdot(\rho D \nabla W) \underbrace{-\rho N \frac{d^{2} W}{d c^{2}}}_{L_{3}}+\underbrace{\rho W \frac{d W}{d c}}_{L_{4}},
$$

where $N \equiv D \nabla c \cdot \nabla c$ is the scalar dissipation rate. The Left Hand Side (LHS) of Eq. (4) involves unsteady and convection terms. The RHS contains a molecular diffusion term and sink/source terms $L_{3}$ and $L_{4}$. The sign of $L_{3}$ or $L_{4}$ is controlled by the sign of $d^{2} W / d c^{2}$ or $d W / d c$ and, therefore, changes with $c$.

First, let us consider an unperturbed laminar flame, i.e., a planar 1D flame that has a steady structure and propagates at a constant speed $S_{L}$ into the unburned gas. Integration of Eq. (4) along the normal to the flame in the coordinate framework attached to it yields

$$
\int_{-\infty}^{\infty}\left(\rho N \frac{d^{2} W}{d c^{2}}-\rho W \frac{d W}{d c}\right) d x=0
$$

Therefore, the terms $L_{3}$ and $L_{4}$ exactly balance one another after integration in this case.

Second, let us consider stretched laminar premixed flames, i.e., spherical or cylindrical flames expanding in quiescent mixture and planar or cylindrical flames stabilized in divergent laminar flows of unburned gas (e.g., $v=g y$ and $u=-g x$ or $u=-g r / 2$ in the planar or cylindrical case, respectively, where $g$ is the rate of strain, $y$-axis is tangential to the flame, $v$ is the $y$-component of velocity vector, and $u$ is the axial, i.e., $x$, or radial, i.e., $r$, flow velocity). As reviewed elsewhere, ${ }^{11,12}$ such flames are used to mimic generic local flame structures in a turbulent flow. As discussed in detail by Dixon-Lewis ${ }^{13}$ and Law, ${ }^{14}$ all these laminar flames can be modeled using the following unsteady $1 \mathrm{D}$ transport equations:

$$
\frac{\partial}{\partial t}(\varrho \Phi)+\varrho g \Phi+\frac{1}{r^{k}} \frac{\partial}{\partial r}\left(r^{k} \varrho v \Phi\right)=\frac{1}{r^{k}} \frac{\partial}{\partial r}\left[r^{k} \varrho d_{\phi} \frac{\partial \Phi}{\partial r}\right]+\varrho S_{\phi} .
$$

Here, $\Phi=\{1, g, c\}, d_{\phi}=\{0, v, D\}, S_{\phi}=\left\{0,-g^{2}+J^{2} / \varrho, \varrho W\right\}, k=\{0,1,2\}$ for planar $(r=x$ in this case), cylindrical, and spherical flames, respectively, with Eq. (6) being valid along a symmetry line $y=0$ if $k<2, \varrho=\rho / \rho_{u}=1 /[1+(\sigma-1) c]$ is the normalized density, $\sigma=\rho_{u} / \rho_{b}$ is the density ratio, $v$ is the kinematic viscosity, subscripts $u$ and $b$ designate fresh mixture and products, respectively, and boundary conditions read

$$
v(0, t)=\frac{\partial c}{\partial r}(0, t)=\frac{\partial g}{\partial r}(0, t)=0, \quad \frac{\partial c}{\partial r}(r \rightarrow \infty, t)=0, \quad g(r \rightarrow \infty, t)=J,
$$

where $J \geq 0$ is an input parameter required to vary the strain rate, with $J=0$ if $k=2$.

Application of a method that was used to derive Eqs. (4)-(6) results in

$$
\frac{\partial}{\partial t}(\varrho W)+\varrho g W+\frac{1}{r^{k}} \frac{\partial}{\partial r}\left(r^{k} \varrho v W\right)=\frac{1}{r^{k}} \frac{\partial}{\partial r}\left[r^{k} \varrho D \frac{\partial W}{\partial r}\right]-\varrho N \frac{d^{2} W}{d c^{2}}+\varrho W \frac{d W}{d c} .
$$

Multiplication of Eq. (8) with $r^{k}$, followed by integration from $r=0$ to $\infty$ yields

$$
\frac{d u_{c}}{d t}+\frac{k u_{c}}{r_{f}} \frac{d r_{f}}{d t}+\frac{1}{r_{f}^{k}} \int_{0}^{\infty} g \varrho W r^{k} d r=\frac{1}{r_{f}^{k}} \int_{0}^{\infty}\left(\varrho W \frac{d W}{d c}-\varrho N \frac{d^{2} W}{d c^{2}}\right) r^{k} d r
$$


where

$$
u_{c}=\frac{1}{r_{f}^{k}} \int_{0}^{\infty} \varrho W r^{k} d r
$$

is the consumption velocity and $r_{f}$ is the flame radius.

The theory of stretched laminar premixed flames ${ }^{15,16}$ is based on the Activation Energy Asymptotic (AEA), i.e., activation temperature of the combustion reaction is considered to be asymptotically high when compared to the temperature of products. Accordingly, $W(c)$ is a highly non-linear function and vanishes everywhere with exception of an asymptotically thin reaction zone at $c \rightarrow 1$. Therefore, variations in the strain rate within such a thin zone can be neglected. Consequently, the third term on the LHS of Eq. (9) reads $g_{f} u_{c}$, where $g_{f}$ is the local value of $g$ in the reaction zone. Moreover, in the case of unity Lewis number, the linear AEA theory of weakly stretched flames ${ }^{15,16}$ predicts that $u_{c}$ is equal to the unperturbed laminar flame speed $S_{L}$. Therefore, substituting the third term on the LHS of Eq. (9) with $g_{f} u_{c}$ and neglecting $d u_{c} / d t$ due to $u_{c} \approx S_{L}$, we arrive at

$$
\frac{1}{r_{f}^{k}} \int_{0}^{\infty}\left(\varrho W \frac{d W}{d c}-\varrho N \frac{d^{2} W}{d c^{2}}\right) r^{k} d r=u_{c}\left(\frac{k}{r_{f}} \frac{d r_{f}}{d t}+g_{f}\right)=u_{c} \dot{s},
$$

where $\dot{s}$ is the stretch rate, which is the major characteristic of weak $\left(\tau_{c} \dot{s} \ll 1\right.$, where $\left.\tau_{c}=D_{u} / S_{L}^{2}\right)$ perturbations of laminar flames within the framework of the AEA theory. ${ }^{15,16}$

Thus, the sum of terms $L_{3}$ and $L_{4}$ on the RHS of Eq. (4), integrated across a stretched laminar flame, is equal to a product of the consumption velocity and stretch rate.

Let us consider turbulent combustion. Ensemble averaging of Eq. (4) yields

$$
\frac{\partial}{\partial t}(\bar{\rho} \tilde{W})+\underbrace{\nabla \cdot \overline{\rho \mathbf{u} W}}_{T_{1}}=\underbrace{\nabla \cdot \overline{\rho D \nabla W}}_{T_{2}} \underbrace{-\overline{\rho N \frac{d^{2} W}{d c^{2}}}}_{T_{3}}+\underbrace{\overline{\rho W \frac{d W}{d c}}}_{T_{4}},
$$

where $\tilde{W} \equiv \overline{\rho q} / \bar{\rho}$ is the Favre-averaged (mass-weighted) value of the rate $W$. Application of Eq. (12) to a statistically planar, 1D flame, followed by integration along $x$, yields

$$
\rho_{u} \frac{d U_{t}}{d t}=\int_{-\infty}^{\infty}\left(\overline{\rho W \frac{d W}{d c}}-\overline{\rho N \frac{d^{2} W}{d c^{2}}}\right) d x,
$$

where

$$
U_{t}=\frac{1}{\rho_{u}} \int_{-\infty}^{\infty} \bar{\rho} \tilde{W} d x
$$

is the turbulent burning velocity. Equation (13) proves that spatially integrated terms $T_{3}$ and $T_{4}$ should not balance one another in the statistically planar, 1D flame. Otherwise, turbulent burning velocity cannot develop, e.g., starting from $U_{t}=S_{L}$ at $t=0$.

However, if the influence of turbulence on a premixed flame is reduced to an increase in the flame surface area by turbulent eddies, whereas perturbations of the local flame structure and burning rate are neglected, as widely assumed for weakly turbulent combustion, then, the RHS of Eq. (13) vanishes. Indeed, taking a mean of $\left(L_{3}+L_{4}\right)$ on the RHS of Eq. (4) consists of averaging this sum over an ensemble of laminar flames in a turbulent flow, i.e., involves local integration of $\left(L_{3}+L_{4}\right)$ along the normal $n$ to each flame element. If the flame element is associated with the unperturbed laminar flame, the result of the local integration vanishes, see Eq. (5). If the ensemble of local integrations is accompanied by integration along $x$ over the mean flame brush, the $\left(L_{3}+L_{4}\right)$ vanishes after this double integration over $x$ and $n$. Therefore, the discussed simplification (unperturbed laminar flames) yields a wrong result, i.e., $d U_{t} / d t=0$. Consequently, the simplification appears to be wrong.

Thus, even unclosed Eq. (12) has allowed us to draw a conclusion regarding a pivotal role played by local perturbations of flame structure actually in weakly turbulent burning. To the best of the present authors' knowledge, this fundamental feature of weakly turbulent premixed combustion has not yet been highlighted in the literature. 
Let us consider the problem of closing Eq. (12). If the probability $\gamma$ of finding finite values of the rate $W$ is low, a characteristic value $W_{f}$ of $W$ within the reaction zone should be high so that a product of $\gamma W_{f}$, normalized using a ratio $\delta_{t} / U_{t}$ of turbulent flame brush thickness to turbulent burning velocity, yields a value of the order of unity. ${ }^{17}$ Indeed, $\gamma W_{f}=O\left(U_{t} / \delta_{t}\right)$ is required in order for $\bar{\rho} \tilde{W}$ to be comparable with the magnitude of the LHS of the Reynolds-averaged Eq. (1). Therefore, one can expect that the magnitudes of terms $T_{3}$ and $T_{4}$ are much (by a factor of $\gamma^{-1}$ ) larger than the magnitudes of other terms in Eq. (12). As reviewed elsewhere, ${ }^{12,18}$ recent experimental data indicate that reaction zones are thin not only in weakly turbulent flows, but also in intense turbulence. Accordingly, terms $T_{3}$ and $T_{4}$ appear to dominate in Eq. (12) in a wide range of conditions.

The fact that two unclosed terms $T_{3}$ and $T_{4}$ dominate in Eq. (12), with $\left(T_{3}+T_{4}\right)$ playing a substantial role, is a challenge. Indeed, in order to propose closure relations for these dominant terms and to predict $\left(T_{3}+T_{4}\right)$, whose magnitude is much less than $\left|T_{3}\right|$ or $\left|T_{4}\right|$, both $T_{3}$ and $T_{4}$ should be modeled with a high precision. A solution could consist of studying the sum of $\left(T_{3}+T_{4}\right)$ instead of modeling each term separately. As the above analysis of laminar flames has shown that the sum of $\left(L_{3}+L_{4}\right)$ in Eq. (4), which is the predecessor of the sum of $\left(T_{3}+T_{4}\right)$ in Eq. (12), yields $u_{c} \dot{s}$ after integration along the normal to a laminar flame, see Eq. (11), we can assume that $\left(T_{3}+T_{4}\right) \propto \gamma u_{c} \dot{s}_{f} / \delta_{r}$, where the reaction zone thickness $\delta_{r}$ results from transformation of the integration over $c$ to the integration along the local normal to the reaction zone. Moreover, it can be expected that $\tilde{W} \propto \gamma W_{f} \propto \gamma u_{c} / \delta_{r}$.

Therefore, we arrive at the following transport equation:

$$
\frac{\partial}{\partial t}(\bar{\rho} \tilde{W})+\nabla \cdot \overline{\rho \boldsymbol{u} W}=\bar{\rho} \tilde{W}\left\langle\dot{s} \mid c_{1}<c<c_{2}\right\rangle .
$$

Here, the stretch rate $\left\langle\dot{s} \mid c_{1}<c<c_{2}\right\rangle$ is conditioned to the reaction zone and, for simplicity, the diffusion term is combined with $\left(L_{3}+L_{4}\right)$ before averaging, because the diffusion term vanishes after integration along the normal to a laminar flame.

In order to assess the proposed model Eq. (15) and to test whether or not $T_{3}$ and $T_{4}$ dominate in Eq. (12), we analyzed data obtained earlier in two sets of Direct Numerical Simulations (DNS), which were consistent with the framework of the present study (single-step chemistry, unity Lewis number). One DNS database (flames H, M, and L) was created by Nishiki et al. ${ }^{19,20}$ by simulating weakly turbulent combustion and was analyzed in a number of recent papers cited elsewhere. ${ }^{21}$ Another DNS database (flames $\mathrm{C}$ and E) was created by Chakraborty et al. ${ }^{22,23}$ by simulating combustion in small-scale intense turbulence (the thin-reaction-zone regime ${ }^{1}$ of premixed burning) and was also analyzed in a number of recent papers cited elsewhere. ${ }^{24}$ Because the DNS data were already discussed in detail in the literature, we will restrict ourselves to a very brief summary of the simulations.

In both cases, unsteady 3D balance equations for mass, momentum, energy, and mass fraction of the deficient reactant were numerically solved and the ideal gas state equation was used. Basic flame characteristics are reported in Table I, where $R e_{t}=u^{\prime} L / v_{u}, D a_{t h}=L S_{L} /\left(u^{\prime} \delta_{t h}\right), K a_{t h}=$ $\left(u^{\prime} / S_{L}\right)^{3 / 2}\left(L / \delta_{t h}\right)^{-1 / 2}$ are the Reynolds, Damköhler, and Karlovitz numbers, respectively, $u^{\prime}$ and $L$ are the rms turbulent velocity and an integral length scale, respectively, and $\delta_{t h}=\left(T_{b}-T_{u}\right) /$ $\max |d T / d x|$ is a laminar flame thickness.

The computational domains were rectangular boxes $\left(8 \times 4 \times 4 \mathrm{~mm}\right.$ or $36.2 \delta_{t h} \times 24.1 \delta_{t h} \times$ $24.1 \delta_{t h}$ in $\mathrm{H}, \mathrm{M}$, and $\mathrm{L}$ or $\mathrm{C}$ and $\mathrm{E}$ DNS, respectively) and were resolved using uniform Cartesian

TABLE I. Flame characteristics.

\begin{tabular}{lcccccc}
\hline \hline Case & $u^{\prime} / S_{L}$ & $L / \delta_{t h}$ & $\sigma$ & $R e_{t}$ & $D a_{t h}$ & $K a_{t h}$ \\
\hline $\mathrm{H}$ & 0.9 & 15.9 & 7.5 & 96 & 18.0 & 0.21 \\
$\mathrm{M}$ & 1.0 & 18.0 & 5.0 & 96 & 17.8 & 0.24 \\
$\mathrm{~L}$ & 1.3 & 21.8 & 2.5 & 96 & 17.3 & 0.30 \\
$\mathrm{C}$ & 7.5 & 2.5 & 5.5 & 48 & 0.33 & 13.0 \\
$\mathrm{E}$ & 11.3 & 3.75 & 5.5 & 110 & 0.33 & 19.5 \\
\hline \hline
\end{tabular}




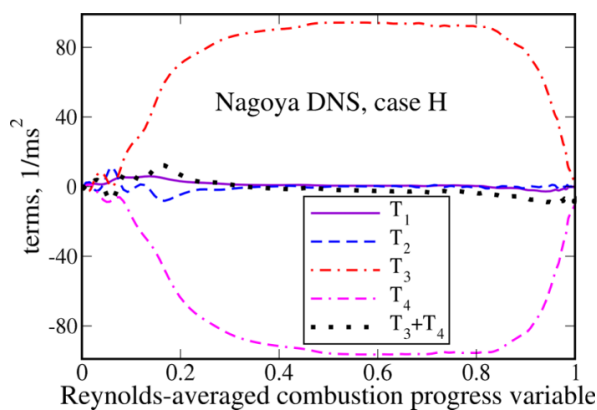

(a)

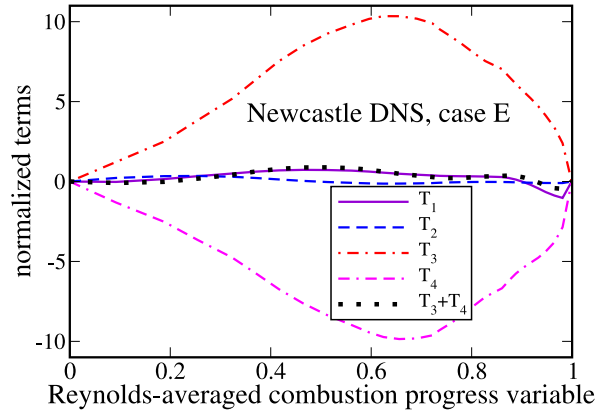

(b)

FIG. 1. Various terms in Eq. (12) obtained from flames (a) $\mathrm{H}$ and (b) E associated with the flamelet and thin-reaction-zone regime, respectively.

meshes of $512 \times 128 \times 128$ or $345 \times 230 \times 230$ points, respectively. The mean flow velocity was parallel to the $x$-axis and normal to the mean flame brush, with the periodic boundary conditions being set at the transverse sides. Homogeneous isotropic turbulence was used to initialize velocity fluctuations and a single planar laminar flame was embedded into the computational domain at $t=0$. In cases $\mathrm{C}$ and $\mathrm{E}$, turbulence decayed with time and averaging was performed over transverse planes at $t / \tau_{t}=3$. In cases $\mathrm{H}, \mathrm{M}$, and $\mathrm{L}$, homogeneous isotropic turbulence was generated in a separate box, was injected into the computational domain at $x=0$, and decayed along the direction $x$. Averaging was performed over transverse $y z$-planes and over time (about 200 snapshots) during that both $U_{t}(t)$ and mean flame brush thickness $\delta_{t}(t)$ oscillated around statistically steady values. ${ }^{21}$

Figure 1 shows that, in line with the above analysis, terms $T_{3}$ and $T_{4}$ dominate not only in weakly turbulent flames, cf. dotted-dashed lines in Fig. 1(a), but also in highly turbulent flames associated with the thin-reaction-zone regime, ${ }^{1}$ see Fig. 1(b).

Figure 2(a) shows that the RHS of Eq. (15) generally reproduces the behavior of the sum of $\left(T_{2}+T_{3}+T_{4}\right)$, extracted from DNSs of highly turbulent flames, but there are quantitative differences. It is worth remembering, however, that, because DNS studies have not yet aimed at analyzing Eq. (12), a mesh used in a typical DNS of turbulent burning may not be sufficiently fine to properly resolve spatial variations in $W d W / d c$ and $N d^{2} W / d c^{2}$. A target-directed DNS with a fine mesh is necessary to quantitatively explore terms $T_{2}, T_{3}$, and $T_{4}$ on the RHS of Eq. (12).

In statistically stationary cases $\mathrm{H}, \mathrm{M}$, and $\mathrm{L}$, the problem can be circumvented by comparing the well-resolved flux $\overline{\rho u W}$ extracted from the DNS with the flux obtained by integrating Eq. (15) whose RHS is extracted from the same DNS. Results are reported in Figure 2(b). Agreement between the DNS data (solid lines) and model results (dashed lines) is encouraging, especially as the model does not involve any tuning constant. Moreover, if $\tilde{W}=\left(\overline{\rho u W}-\overline{\rho u^{\prime \prime} W^{\prime \prime}}\right) /(\bar{\rho} \tilde{u})$, where

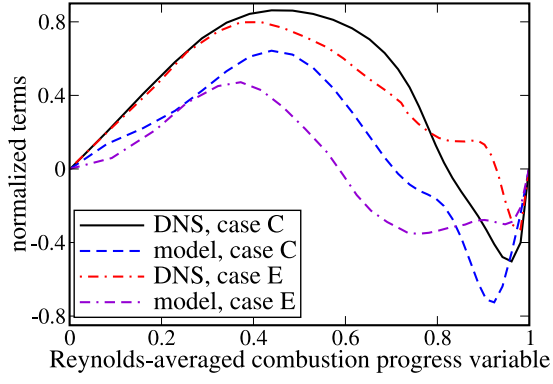

(a)

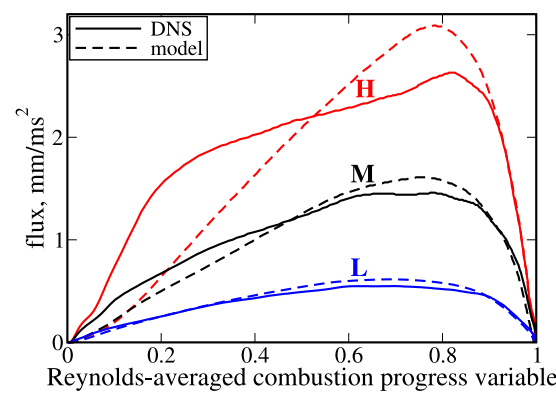

(b)

FIG. 2. (a) Comparison of the sum of $\left(T_{2}+T_{3}+T_{4}\right)$ extracted from DNS and the RHS of Eq. (15) extracted from the same DNS in cases C and E. (b) Comparison of the flux $\overline{\rho u W}$ extracted from DNS data (solid lines) and yielded by Eq. (15) (dashed lines), with $c_{1}$ and $c_{2}$ being set so that $\rho W\left(c_{1}\right)=\rho W\left(c_{2}\right)=\max \{\rho W(c)\} / 2$. 
$\bar{\rho} \tilde{u}=\overline{\rho u}, u^{\prime \prime}=u-\tilde{u}$, and $W^{\prime \prime}=W-\tilde{W}$, is evaluated using (i) $\overline{\rho u W}$ computed by integrating statistically stationary $1 \mathrm{D}$ Eq. (15) and (ii) $\overline{\rho u^{\prime \prime} W^{\prime \prime}}, \bar{\rho}$, and $\tilde{u}$ extracted from the DNS, the spatial integration of the so-obtained $\tilde{W}$ yields $U_{t}=0.97,0.89$, and $0.77 \mathrm{~m} / \mathrm{s}$ in cases $\mathrm{H}, \mathrm{M}$, and L, respectively. These values are close to $U_{t}=1.15,1.02$, and $0.75 \mathrm{~m} / \mathrm{s}$, respectively, extracted straightforwardly from the DNS, thus, supporting the model Eq. (15). Thus, while Eq. (15) does not resolve a problem of closing Eq. (12), as the conditioned stretch rate and $\rho u^{\prime \prime} W^{\prime \prime}$ have still to be modeled, Eq. (15) appears to be a key step forward in the right direction.

Integration of Eq. (15) along $x$ over the mean flame brush yields

$$
\rho_{u} \frac{d U_{t}}{d t}=\int_{0}^{\Lambda_{x}} \bar{\rho} \tilde{W}\left\langle\dot{s} \mid c_{1}<c<c_{2}\right\rangle d x,
$$

where $\Lambda_{x}$ is the length of the computational domain. Figure 3 shows that this model equation reasonably well captures the large-scale dynamics of turbulent burning velocity in flames $\mathrm{H}$ and $\mathrm{L}$ without tuning. It is worth remembering that (i) precision of evaluation of $d U_{t} / d t$ by numerically differentiating $U_{t}(t)$ obtained from the DNS data is limited, because the data were stored once per 100 time steps, and (ii) the highly positive (or negative) values of $d U_{t} / d t$, obtained in the DNS, are associated with growth (or disappearance) of unburned mixture fingers, as discussed elsewhere. ${ }^{21}$ It is also worth remembering that response of a laminar premixed flame to an unsteady stretch rate depends substantially on the frequency of variations in the stretch rate ${ }^{25}$ and such local transient effects can play a role in the interaction of a premixed flame with small-scale turbulent eddies. ${ }^{26}$ The simplest model Eq. (15) does not seem to allow for such small-scale effects, but we are not aware of another model capable of doing so. The issue definitely requires further analysis.

It is worth stressing that Eq. (15) clearly shows an important role played by turbulent stretch rates in premixed combustion and, moreover, implies a crucial role played by the stretch rate $\dot{s}_{l e}$ conditioned to the leading edge $(\bar{c} \rightarrow 0)$ of a premixed turbulent flame brush. Indeed, the structure of Eq. (15) is such that an increase in $\dot{s}_{l e}$ results in increasing $d \tilde{W} / d \bar{c}$ at $\bar{c} \rightarrow 0$ and, therefore, increasing turbulent burning velocity $U_{t} \equiv \rho_{u}^{-1} \int_{-\infty}^{\infty} \bar{\rho} \tilde{W} d x$. This feature of Eq. (15) is consistent with the leading point concept of premixed turbulent combustion, which also highlights the importance of stretch rates conditioned to the leading edge ${ }^{27}$ and is capable of explaining a very strong effect of a decrease in the Lewis number $L e$ on $U_{t}$, which was well documented in various experiments reviewed elsewhere. ${ }^{11,12,26,28}$ Accordingly, extension of the present approach to flames characterized by $L e<1$ appears to be of particular interest in order to model such effects.

In summary, new transport equations for instantaneous and mean reaction rates in a turbulent flow have been derived, see Eqs. (4) and (12), respectively. An analysis of these equations has shown that (i) local perturbations of reaction zone structure by turbulent eddies always play a pivotal role, including weakly turbulent flows, where such effects are often neglected, and (ii) the latter transport equation, i.e., Eq. (12), involves two unclosed dominant terms. A joint closure relation for the sum of these two terms has been developed and validated by processing two sets of DNS data obtained earlier from weakly and highly turbulent premixed flames. The closure relation

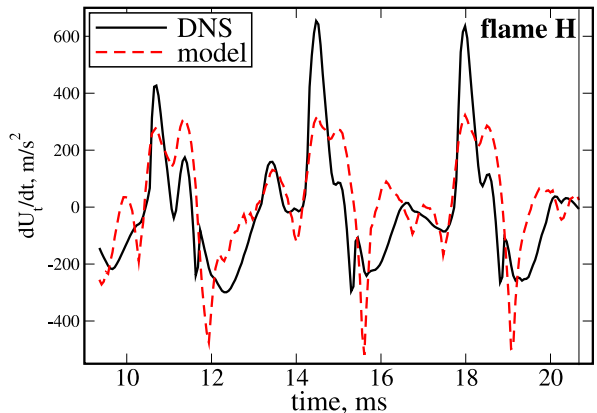

(a)

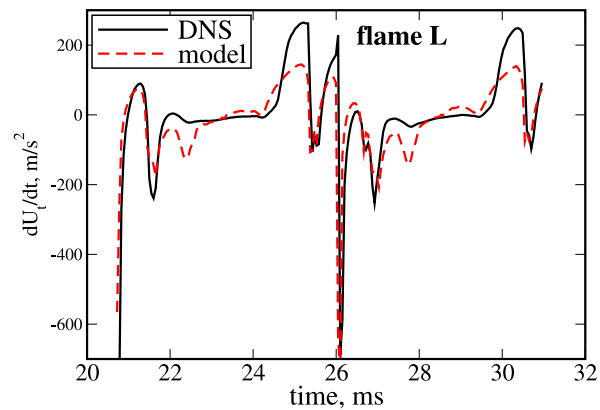

(b)

FIG. 3. Comparison of $d U_{t} / d t$ evaluated by differentiating $U_{t}(t)$ extracted directly from DNS and $d U_{t} / d t$ obtained by integrating 1D Eq. (16) whose RHS is extracted from the same DNS. 
highlights a crucial role played by turbulent stretching in the influence of turbulence on mean reaction rate.

V.A.S. gratefully acknowledges the financial support by ONERA. A.N.L. gratefully acknowledges the financial support by the Chalmers Transport and Energy Areas of Advance, and by the Combustion Engine Research Center (CERC). N.C. gratefully acknowledges the financial support provided by EPSRC, UK.

${ }^{1}$ N. Peters, Turbulent Combustion (Cambridge University Press, Cambridge, UK, 2000).

${ }^{2}$ A. N. Lipatnikov and J. Chomiak, "Turbulent flame speed and thickness: Phenomenology, evaluation, and application in multi-dimensional simulations," Prog. Energy Combust. Sci. 28, 1 (2002).

${ }^{3}$ D. Veynante and L. Vervisch, "Turbulent combustion modeling," Prog. Energy Combust. Sci. 28, 193 (2002)

${ }^{4}$ T. Poinsot and D. Veynante, Theoretical and Numerical Combustion, 2nd ed. (Edwards, Philadelphia, 2005).

${ }^{5}$ R. W. Bilger, S. B. Pope, K. N. C. Bray, and J. F. Driscoll, "Paradigms in turbulent combustion research,” Proc. Combust. Inst. 30, 21 (2005).

${ }^{6}$ N. Chakraborty, M. Champion, A. Mura, and N. Swaminathan, "Scalar-dissipation-rate approach," in Turbulent Premixed Flames, edited by N. Swaminathan and K. N. C. Bray (Cambridge University Press, Cambridge, UK, 2011), pp. 76-102.

${ }^{7}$ R. Borghi, "Turbulent premixed combustion: Further discussions of the scales of fluctuations," Combust. Flame 80, 304 (1990).

${ }^{8}$ S. B. Pope, “The evolution of surface in turbulence,” Int. J. Eng. Sci. 26, 445 (1988).

${ }^{9}$ S. Candel and T. Poinsot, "Flame stretch and the balance equation for the flame area," Combust. Sci. Technol. 170, 1 (1990).

${ }^{10}$ A. Trouvé and T. Poinsot, "Evolution equation for flame surface density in turbulent premixed combustion," J. Fluid Mech. 278, 1 (1994).

${ }^{11}$ A. N. Lipatnikov and J. Chomiak, "Molecular transport effects on turbulent flame propagation and structure," Prog. Energy Combust. Sci. 31, 1 (2005).

12 A. Lipatnikov, Fundamentals of Premixed Turbulent Combustion (CRC Press, Boca Raton, FL, 2012).

${ }^{13}$ G. Dixon-Lewis, "Structure of laminar flames,” Proc. Combust. Inst. 23, 305 (1990).

${ }^{14}$ C. K. Law, Combustion Physics (Cambridge University Press, Cambridge, UK, 2006).

${ }^{15}$ P. Clavin, "Dynamical behavior of premixed flame fronts in laminar and turbulent flows," Prog. Energy Combust. Sci. 11, 1 (1985).

${ }^{16}$ M. Matalon, "Intrinsic flame instabilities in premixed and nonpremixed combustion," Annu. Rev. Fluid Mech. 39, 163 (2007).

${ }^{17}$ P. A. Libby and K. N. C. Bray, "Implications of the laminar flamelet model in premixed turbulent combustion," Combust. Flame 39, 33 (1980).

18 J. F. Driscoll, "Turbulent premixed combustion: Flamelet structure and its effect on turbulent burning velocities," Prog. Energy Combust. Sci. 34, 91 (2008).

${ }^{19}$ S. Nishiki, T. Hasegawa, R. Borghi, and R. Himeno, "Modeling of flame-generated turbulence based on direct numerical simulation databases," Proc. Combust. Inst. 29, 2017 (2002).

${ }^{20}$ S. Nishiki, T. Hasegawa, R. Borghi, and R. Himeno, "Modelling of turbulent scalar flux in turbulent premixed flames based on DNS databases," Combust. Theory Modell. 10, 39 (2006).

${ }^{21}$ A. N. Lipatnikov, J. Chomiak, V. A. Sabelnikov, S. Nishiki, and T. Hasegawa, "Unburned mixture fingers in premixed turbulent flames," Proc. Combust. Inst. 35, 1401 (2015).

${ }^{22}$ N. Chakraborty, G. Hartung, M. Katragadda, and C. F. Kaminski, "Comparison of 2D and 3D density-weighted displacement speed statistics and implications for laser based measurements of flame displacement speed using direct numerical simulation data," Combust. Flame 158, 1372 (2011).

${ }^{23}$ N. Chakraborty and E. R. Hawkes, "Determination of 3D flame surface density variables from 2D measurements: Validation using direct numerical simulation,” Phys. Fluids 23, 065113 (2011).

${ }^{24}$ M. Klein, C. Kasten, Y. Gao, and N. Chakraborty, "A-priori direct numerical simulation assessment of sub-grid scale stress tensor closures for turbulent premixed combustion," Comput. Fluids 122, 1 (2015).

${ }^{25}$ P. Clavin and G. Joulin, "High-frequency response of premixed flames to weak stretch and curvature: A variable density analysis," Combust. Theory Modell. 1, 429 (1997).

${ }^{26}$ P. Venkateswaran, A. Marshall, J. Seitzman, and T. Lieuwen, "Pressure and fuel effects on turbulent consumption speeds of $\mathrm{H}_{2} / \mathrm{CO}$ blends," Proc. Combust. Inst. 34, 1527 (2013).

27 V. R. Kuznetsov and V. A. Sabelnikov, Turbulence and Combustion (Hemisphere Publishing Corporation, New York, 1990).

${ }^{28}$ P. Venkateswaran, A. Marshall, J. Seitzman, and T. Lieuwen, "Scaling turbulent flame speeds of negative Markstein length fuel blends using leading points concepts," Combust. Flame 162, 375 (2015). 